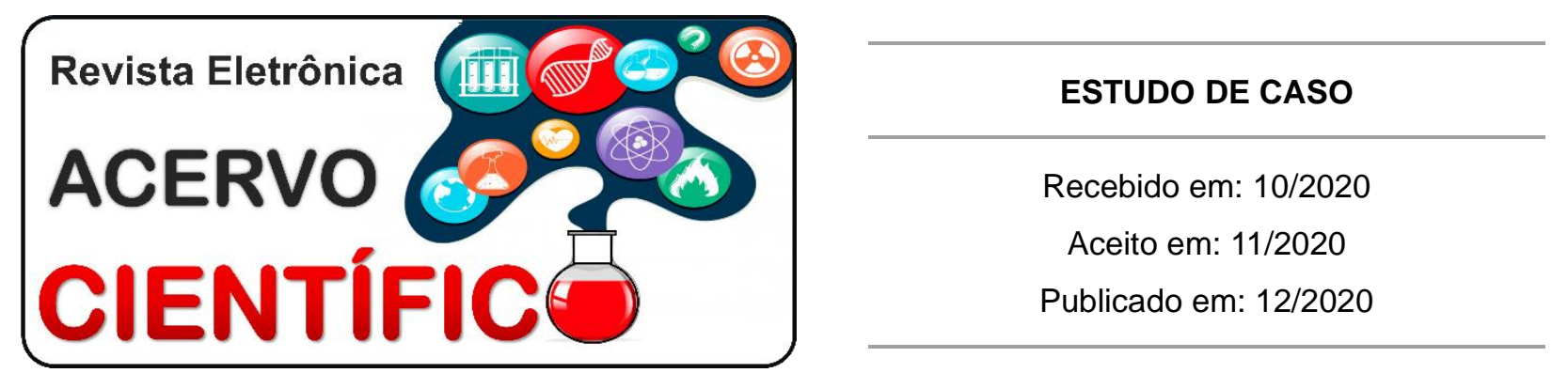

\title{
Delirium Tremens: revisão de aspectos principais em um relato de caso
}

\author{
Delirium Tremens: review of main aspects in a case report
}

Delirium Tremens: revisión de los principales aspectos de un reporte de caso

Guilherme Pitol1*, Rafaela Paulino1, Lucas Rodrigues Mostardeiro1, Bruna Chaigar Venzke ${ }^{1}$, Acauã Ferreira da Cunha ${ }^{1}$, Bruna Eliza Trevisan Dal'Osto ${ }^{1}$, Gabriela Tonini Maciel ${ }^{1}$, Bibiana Bauer Barcellos ${ }^{1}$, Ana Laura Tonon de Quadros ${ }^{1}$, Daniela Fredi Santi ${ }^{1}$.

Resumo: Esse artigo buscou relatar um caso de Delirium Tremens, um caso raro na medicina geral e pouco comum em indivíduos tratados para abstinência alcoólica, mas que possui grande importância em questão de morbimortalidade. $O$ trabalho avalia um atendimento em um hospital psiquiátrico com um desfecho favorável e disserta sobre estratégias valorosas para um bom prosseguimento em casos semelhantes que médicos e equipes de saúde possam vir a experienciar. Trata-se de um paciente masculino de 57 anos que fazia uso abusivo de bebidas alcoólicas por 13 anos que apresentou condutas hetero e autoagressivas, sendo então trazido por familiares a um hospital psiquiátrico. Foi manejado inicialmente bem, porém evoluiu com alteração do ciclo sono-vigília, tremores, alteração da consciência e alterações da sensopercepção. Foi medicado e evoluiu com melhora gradual da sintomatologia, sem necessidade de ser internado em hospital clínico. O relato desse caso é importante para a rememoração de um quadro importante, pouquíssimo frequente na medicina em geral, mas que é relevante para a prática em urgência e emergência.

Palavras-chave: Transtornos neurocognitivos, Neurologia, Delirium Tremens.

Abstract: This article sought to report a case of Delirium Tremens, a rare case in general medicine and uncommon in individuals treated for alcohol withdrawal, but which has great importance in terms of morbidity and mortality. The work evaluates a service in a psychiatric hospital with a favorable outcome and talks about valuable strategies for good progress in similar cases that doctors and health teams may experience. This is a 57-year-old male patient who had been abusing alcoholic beverages for 13 years, who had hetero and self-aggressive behaviors, and was then brought by family members to a psychiatric hospital. It was initially well managed, but it evolved with changes in the sleep-wake cycle, tremors, changes in consciousness and changes in perception. He was medicated and progressed with a gradual improvement in symptoms, without the need to be admitted to a clinical hospital. The report of this case is important for the remembrance of an important condition, which is very rare in medicine in general, but which is relevant for urgent and emergency practice.

Keywords: Neurocognitive disorders, Neurology, Delirium Tremens.

Resumen: Este artículo buscó reportar un caso de Delirium Tremens, un caso raro en medicina general y poco común en individuos tratados por abstinencia alcohólica, pero que tiene gran importancia en términos de morbilidad y mortalidad. El trabajo evalúa un servicio en un hospital psiquiátrico con resultado favorable y

1 Universidade Católica de Pelotas (UCPel), Pelotas - RS. *E-mail: md.guilhermepitol@gmail.com 
habla de estrategias valiosas para el buen avance en casos similares que puedan experimentar médicos y equipos de salud. Se trata de un paciente masculino de 57 años que había estado abusando de bebidas alcohólicas durante 13 años, que tenía comportamientos hetero y autoagresivos, y luego fue llevado por familiares a un hospital psiquiátrico. Inicialmente se manejó bien, pero evolucionó con cambios en el ciclo sueño-vigilia, temblores, cambios en la conciencia y cambios en la percepción. Fue medicado y progresó con una mejora progresiva de los síntomas, sin necesidad de ser ingresado en un hospital clínico. El reporte de este caso es importante para el recuerdo de una condición importante, que es muy rara en la medicina en general, pero que es relevante para la práctica urgente y de emergencia.

Palabras clave: Trastornos neurocognitivos, Neurología, Delirium Tremens.

\section{INTRODUÇÃO}

Estado Confusional Agudo (Delirium) é caracterizado como uma síndrome mental orgânica em que há alterações importantes principalmente no nível de consciência e na orientação, tendo então interesse neuropsiquiátrico. Contudo, especificamente nos pacientes em tratamento de dependência alcoólica, um tipo de delirium chamado Delirium Tremens é especialmente importante. Estima-se que seja uma afecção rara, se apresentando em cerca de 3 a $5 \%$ de pacientes que necessitam de hospitalização para tratamento da dependência ao álcool; porém, esta é a principal causa de morbimortalidade nesse grupo de pacientes, em que índices de mortalidades estão entre 1 a 15\% (SCHUCKIT MA, 2014; KAFLE P, et al., 2019).

O Delirium Tremens (DT) se caracteriza por apresentar sintomatologia de um Estado Confusional Agudo, porém ocorre em pacientes dependentes ao álcool, se apresenta nos dias que se sucedem a cessão da ingesta alcoólica e há também sintomas de disfunção autonômica como tremores (daí o nome), febre, hipertensão e taquicardia. Em particular, a presença de alucinações visuais e táteis com insetos é comum (MALDONADO JR, 2017). Os fatores de risco conhecidos para a síndrome são o prévio acometimento, alterações em eletrólitos e plaquetas, hiperhomocisteinemia, infecções em curso e alterações em enzimas hepáticas; além disso, também são estudados que idade avançada e baixos níveis de magnésio sérico estão associados com maior severidade do quadro (GROVER S e GOSH A, 2018).

A respeito do tratamento da afecção, o objetivo é reduzir a severidade dos sintomas adrenérgicos e restaurar qualquer parâmetro clínico que possa ter sido comprometido. A primeira escolha para os casos de DT são os benzodiazepínicos, que já possuem vasta literatura que apoia o uso. O prognóstico dos pacientes é extremamente variado e depende das doenças de base (SCHMIDT KJ, et al., 2016; HUI D, 2018; LONG D, et al., 2017).

Então, o objetivo desse estudo é relatar o caso de um paciente dependente de álcool sendo acompanhado em um hospital psiquiátrico que desenvolveu Delirium Tremens durante a internação para desintoxicação.

\section{RELATO DE CASO}

Homem, branco, 57 anos, divorciado, ensino superior completo, hipertenso e etilista há 13 anos com consumo de "fardos de cerveja" diariamente, mora com a mãe de 81 anos, internado em hospital psiquiátrico devido a quadro de heteroagressividade e expondo-se a riscos. Naquele momento não aderente a tratamento ambulatorial, foi trazido após 5 dias internado em hospital particular para manejo de quadro clínico agudo. Apresentava-se emagrecido e confuso, disse estar sob efeito de álcool, negou uso atual ou prévio de qualquer outro tipo de droga. No exame mental e físico, com roupas adequadas ao ambiente, porém higiene não adequada; lúcido, hipovigil e normotenaz; não apresentava alterações na sensopercepção; desorientado em tempo e espaço, memória imediata e recente alteradas, afeto hipomodulado; pensamento predominantemente mágico, curso agregado, conteúdo com delírio de grandeza e velocidade normal, bradilálico e com juízo crítico parcialmente comprometido. Estava em uso de Diazepam, Haloperidol, Tiamina e Complexo B, captopril e Furosemida. Apresentava pressão arterial de 
160x100 mmHg, Hemoglico teste (HGT) de $85 \mathrm{mg} / \mathrm{dL}$, frequência cardíaca de 104 batimentos por minuto, temperatura axilar de $36,6^{\circ} \mathrm{C}$. Restante do exame físico não digno de nota. No quarto dia de internação, paciente apresenta alteração no ciclo sono-vigília, rebaixamento do sensório, febre de $38,6^{\circ} \mathrm{C}$ e tremores. Ao exame do estado mental, relata ver baratas subindo pelas paredes do quarto e sente formigas subindo nas suas pernas.

Além disso, observa-se que o paciente apresenta Sinal de Sampaio presente: o examinador entrega um fio imaginário ao doente, esse o pega e quando ordenado que faça um nó com tal fio imaginário, realiza-o. Ao exame físico, frequência cardíaca de 108 batimentos por minuto e pressão arterial de 140x90 mmHg. Foi então medicado imediatamente com $20 \mathrm{mg}$ de Diazepam via oral (VO) e prescrito mais $10 \mathrm{mg}$ a cada 8 horas. O paciente evoluiu nos dias seguintes com melhora do nível de consciência e sem novas alterações clínicas. O manejo realizado foi satisfatório, com parâmetros laboratoriais e clínicos estabilizados e sem piora do quadro, assim não sendo necessário encaminhamento para hospital geral de referência.

\section{DISCUSSÃO}

Delirium Tremens (DT) é uma patologia associada à dependência ao álcool e pode acontecer durante a desintoxicação dessa substância. Trata-se de uma alteração principalmente da consciência e sensopercepção associadas a uma disfunção autonômica. É uma entidade conhecida há mais de 200 anos e teve um período relatado de 100 anos sem alteração na mortalidade, que ia de 5 a $25 \%$ em pacientes com DT. Novos dados na literatura, entretanto, mostram que nos últimos 14 anos a mortalidade para Delirium Tremens foi impactada positivamente: apesar de dado ainda importante, atualmente a mortalidade se aproxima de 1 a 15\% nos pacientes hospitalizados com a afecção (SCHUCKIT MA, 2014; KAFLE P, et al., 2019).

O diagnóstico dessa doença é firmado pelo Manual Diagnóstico e Estatístico de Transtornos Mentais, o DSM-5 (2014), naquele paciente que apresenta tanto critérios para abstinência alcoólica, quanto para Delirium. Ou seja, em pacientes que diminuíram ou cessaram o uso crônico de álcool, dois dos seguintes sintomas: hiperatividade autonômica, tremor em mãos, insônia, náusea ou vômitos, alucinações ou ilusões transitórias, agitação psicomotora, ansiedade ou convulsões clônico-tônicas generalizadas; além disso, preenchem: perturbação da atenção e da consciência, com oscilação ao longo de um dia tanto na gravidade quanto no período de tempo da alteração, perturbação da cognição e sendo tais sintomas não mais bem explicados por condição neurocognitiva preexistente ou simultânea. No nosso relato de caso, o paciente tinha 5 critérios para abstinência alcoólica e preenchia todos os critérios para Delirium.

O início da sintomatologia se apresenta após 3 a 5 dias sem ingesta alcoólica e persiste por até 8 dias após (SCHUCKIT MA, 2014). Há um instrumento de medida que é realizado principalmente na conduta perante o quadro clínico, a escala Clinical Institute Withdrawal Assessment for Alcohol, Revised (CIWA-Ar). No consenso brasileiro de Psiquiatria sobre a Síndrome de Abstinência do Álcool, a pontuação dessa escala que avalia os critérios clínicos como náusea, tremor, sudorese, alterações da sensopercepção, inquietação e alteração de orientação servem como triagem à escolha do nível de atendimento. No nosso relato, o paciente estava em nível grave de Delirium Tremens, então sendo necessária a internação em hospital integral. Como já estava nesse nível de atendimento e não houve deterioração do quadro, não foi necessária mudança hospitalar.

Pelo início gradual, importância do quadro e possível necessidade de internação hospitalar, anamnese, exames físico e mental devem ser minuciosos em todos os casos de Delirium, principalmente no relacionado à abstinência alcoólica. Inicialmente, o paciente pode não estar em um hospital psiquiátrico e, inclusive, pode estar em atendimento ambulatorial. A história médica pregressa deve ser bem conhecida do médico e da equipe que estiverem sob tutela do doente, mesmo em situações de urgência e emergência: histórico de doenças neurológicas, como epilepsia, acidente vascular cerebral, doença de Parkinson, ou uso de medicações antipsicóticas de primeira geração como haldol para pesquisa de para efeitos como a síndrome extrapiramidal são importantes diagnósticos diferenciais. Saber o abuso de álcool é imprescindível para o diagnóstico correto, porém a idade do paciente também é importante para diferenciar diagnósticos: 0 
estado confusional agudo costuma acontecer com frequência em idosos por infecções urinárias ou pneumonias, e não está relacionado com o álcool (LONG D, et al., 2017). No exame físico, mesmo que o paciente não esteja colaborativo, a avaliação de sinais vitais, hidratação, condição da pele e possíveis locais com focos de infecção devem ser sempre pesquisados. Em pacientes que já chegam ao hospital em depressão do sensório importante, a avaliação fenotípica se demonstra imprescindível; por exemplo, o hálito cetônico, urêmico ou alcoólico, associado com fácies renal, icterícia ou paciente jovem e magro, podem estar relacionados a cetoacidose diabética, falência renal, encefalopatia hepática ou abuso de álcool.

Observar a língua do paciente e procurar por mordidas, ou se houver fratura/deslocamento do ombro também é valoroso, pois são sinais presuntivos de convulsão; observação de pupilas também é essencial para procurar por intoxicação com drogas simpaticomiméticas. Um exame neurológico completo deve ser realizado a fim de descartar hipóteses agudas como um acidente vascular encefálico; testar os pares cranianos, observar se há alteração de rima palpebral, desvio da comissura labial, alterações desde paralisia até a lentidão na resposta ocular a um estimulo luminoso, sinal de roda denteada, sinal do canivete, flapping, clônus, nistagmo, alterações da marcha são todos exemplos de exames que não devem ser esquecidos na hora de uma avaliação completa.

Os autores reforçam que a ausência de sinal focal não exclui a possibilidade de lesão aguda no sistema nervoso central, e em casos de ausência de motivo óbvio de uma causa de Delirium, um exame de neuroimagem, punção lombar ou eletroencefalograma devem ser realizados (FRANCIS J e YOUNG B, 2020). Além disso, o exame do estado mental deve se ater a principalmente a avaliação da consciência, orientação, sensopercepção e atenção. Esses quatro pilares estão mais relacionados a síndromes orgânicas do que a síndromes psiquiátricas, portanto quando alterados, a parte clínica do paciente deve ser primariamente investigada (KAFLE $P$, et al., 2019).

Delirium Tremens é uma das doenças em que a participação do familiar ou acompanhante é imprescindível para a decisão diagnóstica e terapêutica. No caso retratado, o paciente foi trazido ao hospital psiquiátrico pela ex-esposa, que orientou os médicos e que foi a responsável pela anamnese do doente, visto que ele não estava em condições e nem cooperava com os médicos e a equipe de saúde. Em casos que o paciente está com o sensório deprimido, nota-se ainda mais a importância de um acompanhante com bom conhecimento do doente que será investigado.

Fatores de risco para a enfermidade são o abuso crônico de álcool, histórico de convulsões por abstinência, já ter tido Delirium Tremens anteriormente, ter mais de 30 anos de idade, ter outras doenças ativas, ter abstinência mesmo com altos níveis de álcool no sangue e demora de mais de 2 dias para apresentar abstinência após última ingesta de álcool (HOFFMAN RS e WEINHOUSE GL, 2020). No relato, o paciente apresentava três desses fatores de risco: estava internado durante 5 dias para tratamento de doença clínica e logo foi internado para desintoxicação; tinha idade maior que 30 anos e ingeria álcool de maneira crônica e sustentada.

Em relação ao tratamento, a literatura atual recomenda uso de benzodiazepínicos de maneira profilática apenas para pacientes com alto risco de desenvolver abstinência e suas variantes, sendo escolha de Diazepam ou Cloridrato de Clordiazepóxido para pacientes sem alterações hepáticas, e Lorazepam ou Oxazepam para aqueles com cirrose ou hepatite alcoólica aguda. Se não for alto risco, os autores recomendam o tratamento apenas na presença de sintomatologia, com as mesmas escolhas de medicamentos. Em ambos os casos, Diazepam 5 a $10 \mathrm{mg}$ intravascular (IV) ou Lorazepam 2 a 4mg IV em pacientes com problemas hepáticos poderiam ser a escolha terapêutica. O paciente deve ser revisto a cada 10 a 15 minutos para revisão da efetividade e reavaliação para novo uso da medicação IV.

Em casos graves, os autores comentam que até doses massivas de Diazepam como mais de $500 \mathrm{mg}$ IV são necessárias para controle inicial de sintomas (HOFFMAN RS e WEINHOUSE GL, 2020). Por via oral, a literatura demonstra que nos casos de sintomas leves de abstinência o Diazepam pode ser usado até 50mg a cada 6 a 12 horas no primeiro dia, seguindo com $25 \mathrm{mg}$ a cada 6 horas no segundo dia, $25 \mathrm{mg}$ a cada $12 \mathrm{~h}$ no terceiro e finalizando o quarto dia com $25 \mathrm{mg}$ à noite, fixo. Caso não haja remissão, a terapêutica fica a 
critério médico, sendo os benzodiazepínicos a principal indicação (HOLT SR e TETRAULT JM, 2020; HUI D, 2018). Um empecilho que temos em nosso país é que nem todas as opções terapêuticas atuais estão disponíveis, principalmente nos serviços de emergência que são financiados por verba pública. Em nosso relato, tínhamos à disposição apenas a formulação do Diazepam, na forma oral ou intramuscular (IM). Optamos por um esquema terapêutico um pouco mais antigo, com dose máxima de 40mg oral diária, visto que no hospital psiquiátrico não havia equipe de plantão clínico caso necessidade.

Não foi provado ainda que profilaxia com outras medicações que não os benzodiazepínicos seja efetiva para prevenir Delirium Tremens; mesmo assim, Gabapentina e Carbamazepina têm efeitos similares na diminuição de sintomas de abstinência alcoólica e são usados frequentemente. Os autores mais atuais não recomendam uso de antipsicóticos ou anticonvulsivantes como tratamento enquanto em vigência de DT; a reposição de vitaminas, especialmente o complexo $B$, é indicado pelos autores na vigência de DT (HOFFMAN RS e WINEHOUSE GL, 2020; HOLT SR e TETRAULT JM, 2020; MAINEROVA B, et al., 2015; MIRIJELLO A, et al., 2015). No momento do relato, o paciente estava em uso contínuo de Haloperidol 10mg diários. Não foram vistos efeitos indesejados.

As informações sobre prognóstico de pacientes com Delirium Tremens na literatura mundial são escassas. É esperado que o paciente trate as causas base que levaram à apresentação clínica, ou seja, cessação da ingesta alcoólica, tratamento de infecções, correção de alterações laboratoriais são importantes medidas para assegurar melhor qualidade de vida e prevenir recorrência. Já foi discutido que o próprio diagnóstico de DT é fator de risco para sua ocorrência, então tratar fatores mutáveis deve ser o principal foco do médico assistente (GROVER S e GHOSH A, 2018; SALOTTO K, et al., 2017; WEISELBERG R, et al., 2011).

Consideramos que uma escala prognóstica poderia ser de grande valia para o tratamento adequado e guiado por evidências, visto que carecemos de uma ferramenta desse tipo no cenário atual. Por exemplo, o estudo de revisão sistemática guiado por Lindroth $\mathrm{H}$ et al. (2018) nos alertou que as escalas prognósticas que avaliam o Delirium, síndrome, demonstram inadequadas capacidades de previsões. Portanto, é natural que ao invés de avaliar uma ampla gama de afecções, uma escala que avaliasse pontualmente o Delirium associado à retirada do álcool poderia ter melhor aplicabilidade para esses casos.

\section{CONSIDERAÇÕES FINAIS}

Delirium Tremens é uma entidade clínica importante de ser estudada, especialmente para médicos e equipes de saúde que estão em contato com pacientes em luta contra a dependência alcoólica e/ou hospitais de urgência ou emergência. É uma afecção extremamente rara na população em geral, e pouco frequente na população específica, porém é de extrema relevância visto que é a mais importante causa de mortalidade em indivíduos abstinentes de álcool. A categoria relato de caso é uma maneira interessante de trazer esse assunto a ser discutido, apesar de que estudos com maior número de pacientes são importantes para mudanças maiores no tratamento e manejo dessa enfermidade.

\section{REFERÊNCIAS}

1. FRANCIS J, YOUNG B. Diagnosis of delirium and confusional states. UpToDate, 2020.

2. GROVER S, GHOSH A. Delirium Tremens (DT): Assessment and management. Journal of Clinical and Experimental Hepatology, 2018; 8(4): 460-470.

3. HOFFMAN RS, WEINHOUSE GL. Management of moderate and severe alcohol withdrawal syndromes. UpToDate, 2020.

4. HOLT SR, TETRAULT JM. Ambulatory management of alcohol withdrawal. UpToDate, 2020.

5. HUI D. Benzodiazepines for agitation in patients with delirium. Current Opinion in Supportive and Palliative Care, 2018; 12(4): 489-494.

6. KAFLE P, et al. Twenty-Eight-Day-Long Delirium Tremens. Journal of Investigative Medicine High Impact Case Reports, 2019; 7.

7. LONG D, et al. The emergency medicine management of severe alcohol withdrawal. The American Journal of Emergency Medicine, 2017; 35(7): 1005-1011. 
8. LINDROTH H, et al. Systematic review of prediction models for delirium in the older adult inpatient. BMJ Open, 2018; 8(4).

9. MAINEROVA B, et al. Alcohol withdrawal delirium - diagnosis, course and treatment. Biomedical Papers of the Medical Faculty of the University Palacky, 2015; 159(1):44-52.

10. MANUAL DIAGNÓSTICO E ESTATÍSTICO DE TRANSTORNOS MENTAIS: DSM-5. Porto Alegre: Artmed, 2014; $947 \mathrm{p}$.

11. MALDONADO JR. Novel Algorithms for the Prophylaxis and Management of Alcohol Withdrawal SyndromesBeyond Benzodiazepines. Critical Care Clinics, 2017; 33(3): 559-599.

12. MIRIJELLO A, et al. Identification and Management of Alcohol Withdrawal Syndrome. Drugs, 2015; 75(4): 353-365.

13. SALOTTOLO K, et al. Occurrence, Predictors, and Prognosis of Alcohol Withdrawal Syndrome and Delirium Tremens Following Traumatic Injury. Critical Care Medicine, 2017; 45(5): 867-874.

14. SCHUCKIT MA. Recognition and Management of Withdrawal Delirium (Delirium Tremens). New England Journal of Medicine, 2014; 371(22): 2109-2113.

15. SCHMIDT KJ, et al. A Review of the Treatment of Severe Alcohol Withdrawal. Annals of Pharmacotherapy, 2016; 50(5): 389-401.

16. WEISELBERG RS, et al. Predicting Delirium Tremens. The Journal of Trauma: Injury, Infection, and Critical Care, $2011 ; 70(1): 263$. 